\title{
In vitro dimerization of human immunodeficiency virus type 1 (HIV-1) spliced RNAs
}

\author{
LUCILE SINCK, ${ }^{1}$ DELPHINE RICHER, ${ }^{1}$ JANE HOWARD, ${ }^{2}$ MARINA ALEXANDER, ${ }^{2}$ DAMIAN F.J. PURCELL, ${ }^{2}$ \\ ROLAND MARQUET, ${ }^{1}$ and JEAN-CHRISTOPHE PAILLART ${ }^{1}$ \\ ${ }^{1}$ Architecture et Réactivité de l'ARN, Université Louis Pasteur, CNRS, IBMC, 67084, Strasbourg cedex, France \\ ${ }^{2}$ Department of Microbiology and Immunology, University of Melbourne, Parkville, Victoria 3010, Australia
}

\begin{abstract}
The human immunodeficiency virus type 1 (HIV-1) packages its genomic RNA as a dimer of homologous RNA molecules that has to be selected among a multitude of cellular and viral RNAs. Interestingly, spliced viral mRNAs are packaged into viral particles with a relatively low efficiency despite the fact that they contain most of the extended packaging signal found in the 5' untranslated region of the genomic RNA, including the dimerization initiation site (DIS). As a consequence, HIV-1 spliced viral RNAs can theoretically homodimerize and heterodimerize with the genomic RNA, and thus they should directly compete with genomic RNA for packaging. To shed light on this issue, we investigated for the first time the in vitro dimerization properties of spliced HIV-1 RNAs. We found that singly spliced (env, vpr) and multispliced (tat, rev, and nef) RNA fragments are able to dimerize in vitro, and to efficiently form heterodimers with genomic RNA. Chemical probing experiments and inhibition of RNA dimerization by an antisense oligonucleotide directed against the DIS indicated that the DIS is structurally functional in spliced HIV-1 RNA, and that RNA dimerization occurs through a loop-loop interaction. In addition, by combining in vitro transcription and dimerization assays, we show that heterodimers can be efficiently formed only when the two RNA fragments are synthesized simultaneously, in the same environment. Together, our results support a model in which RNA dimerization would occur during transcription in the nucleus and could thus play a major role in splicing, transport, and localization of HIV-1 RNA.
\end{abstract}

Keywords: HIV-1; RNA dimerization; mRNA; transcription; packaging selection

\section{INTRODUCTION}

The genome of retroviruses, including the human immunodeficiency virus type 1 (HIV-1), is packaged as two homologous (+) strand RNA molecules noncovalently associated close to their $5^{\prime}$-end in a region called the dimer linkage structure (DLS) (Bender and Davidson 1976; Murti et al. 1981; Paillart et al. 2004b). Dimerization of retroviral genomic RNA is an ubiquitous process, and in vitro experiments with genomic RNA fragments have shown that HIV-1 RNA dimerization takes place in the $5^{\prime}$ untranslated region $\left(5^{\prime}\right.$-UTR), at the dimerization initiation site (DIS) (Fig. 1A; Laughrea and Jetté 1994; Skripkin et al. 1994; Muriaux et al. 1995). The DIS, a highly

Reprint requests to: Jean-Christophe Paillart, Architecture et Réactivité de l'ARN, Université Louis Pasteur, CNRS, IBMC, 67084, Strasbourg cedex, France; e-mail: jc.paillart@ibmc.u-strasbg.fr; fax: $(+33)(0) 38860$ 22 18; or Roland Marquet, Architecture et Réactivité de l'ARN, Université Louis Pasteur, CNRS, IBMC, 67084, Strasbourg cedex, France; e-mail: r.marquet@ibmc.u-strasbg.fr; fax: (+33) (0)3 88602218.

Article published online ahead of print. Article and publication date are at http://www.rnajournal.org/cgi/doi/10.1261/rna.678307. conserved sequence located upstream of the major splice donor (SD) site, folds into a hairpin structure with a 9 nucleotide (nt) loop containing a 6 nt self-complementary sequence (Laughrea and Jetté 1994; Paillart et al. 1994; Skripkin et al. 1994; Muriaux et al. 1995). Dimerization of HIV-1 RNA is initiated at the self-complementary sequence of each RNA monomer via an intermolecular kissing-loop interaction (Laughrea and Jetté 1994; Paillart et al. 1994; Skripkin et al. 1994; Muriaux et al. 1995; Clever et al. 1996; Haddrick et al. 1996). In vitro, this kissing-loop complex can be converted into a more stable dimer (also called a tight dimer) by the viral nucleocapsid protein at $37^{\circ} \mathrm{C}$ or by incubation at high temperature $\left(55^{\circ} \mathrm{C}\right)$ (Laughrea and Jette 1996; Muriaux et al. 1996; Takahashi et al. 2000). Stabilization of the RNA dimer (mature dimer) in virions has been observed upon proteolytic cleavage of Gag for several retroviruses (Oertle and Spahr 1990; Stewart et al. 1990; Fu and Rein 1993; Fu et al. 1994) and retrotransposons (Feng et al. 2000). However, whether the stabilization of the RNA dimer observed in vitro and ex vivo corresponds to the same molecular mechanism remains unclear (for review, see Paillart et al. 2004b). 
A

$\underbrace{\text { PolyA }}_{\text {TAR }}$
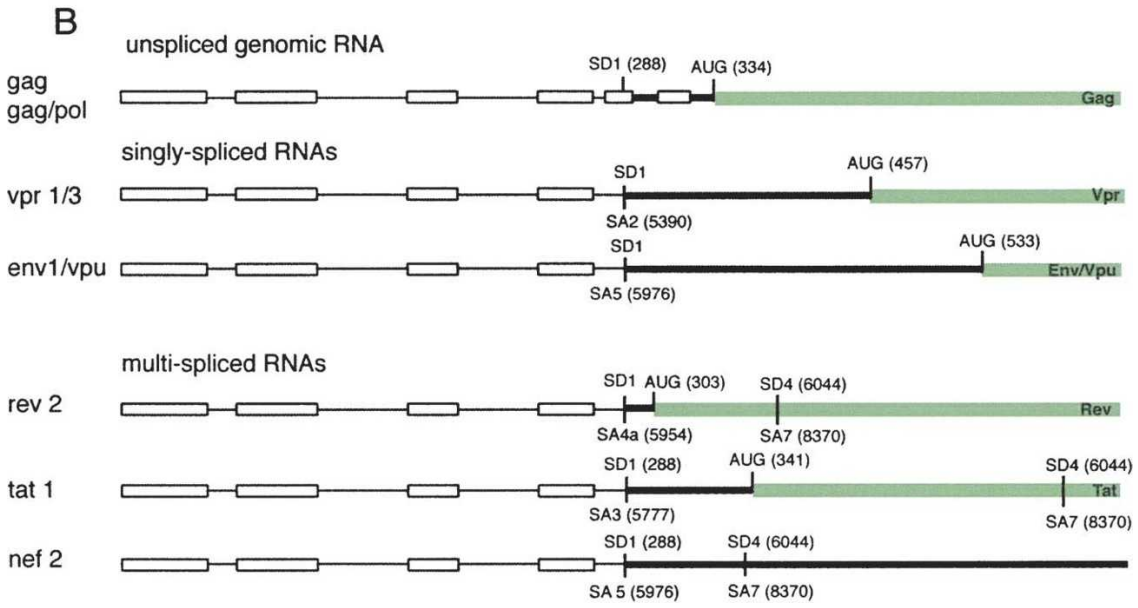

FIGURE 1. Genomic and spliced HIV-1 (NL4.3) RNAs. (A) Scheme of the first $600 \mathrm{nt}$ of HIV-1 genomic RNA. (R) repeat sequence, (TAR) trans-acting responsive element, (polyA) 5' copy of the polyadenylation signal, (U5) unique sequence at the $5^{\prime}$ end of the RNA genome, (PBS) primer-binding site, (DIS) dimerization initiation site, (SD) major splice-donor site, (PSI) major packaging signal, and (AUG) translation initiator codon of gag gene. (B) Spliced and genomic RNAs used in this study. (Open boxes) The different RNA elements, (black thick lines) untranslated regions, and (green thick lines) coding regions. The positions of splice donor (SD) and acceptor (SA) sites are indicated above and under the genomic RNA, respectively.

Genomic RNA dimerization is crucial for retroviral replication. Indeed, alterations of the DIS strongly affect viral replication by perturbing two essential steps of the viral cycle: packaging of the genomic RNA and reverse transcription (Berkhout and van Wamel 1996; Haddrick et al. 1996; Paillart et al. 1996a; Laughrea et al. 1997; Shen et al. 2000; Houzet et al. 2007). Studies using chimerical vectors or synthetic RNA fragments demonstrated that the DIS also plays a major role in HIV-1 recombination (Balakrishnan et al. 2001, 2003; Andersen et al. 2003; Chin et al. 2005). Overall, these results suggest that dimerization is a prerequisite for RNA packaging and a key process for virus evolution (Russell et al. 2004).

However, while the mechanism of in vitro RNA dimerization and its possible roles in the replication cycle have been extensively studied, the place where RNA dimerization takes place (nucleus versus cytoplasm or cellular membrane) is still a point of discussion (Russell et al. 2004). Indeed, after their synthesis in the nucleus of the host cell, HIV-1 RNAs have several fates. Contrary to the vast majority of cellular pre-messenger RNAs, which are completely spliced, approximately half of the HIV-1 RNA avoids the splicing reaction and is directly transported to the cytoplasm (Stoltzfus and Madsen 2006). The unspliced RNA is used as mRNA for the synthesis of the Gag and
Gag-Pol proteins and as genomic RNA (gRNA) that is encapsidated in the viral particle in its dimeric form (Swanson and Malim 2006). During the packaging process, gRNA must be preferentially selected among a multitude of cellular and viral mRNAs. The general RNA packaging mechanism developed by retroviruses involves cis elements present on the viral RNA and trans-acting factor(s) encoded by the viral genome (Lever 2000; D'Souza and Summers 2005). In HIV-1, a major packaging signal (Psi or $\Psi$ ) has been located in the 5' UTR, between the SD site and the AUG start codon of Gag (Fig. 1A). It folds into a stem-loop structure (SL3) whose deletion profoundly affected viral replication by reducing the yield of gRNA present in the viral particles (Lever et al. 1989; Aldovini and Young 1990; Clavel and Orenstein 1990). However, the packaging specificity is not restricted to this region, although Psi is uniquely present on gRNA (Fig. 1A). Several regions upstream of the SD site (including TAR, the polyA, the PBS, and the DIS) and downstream of the translation initiation codon of Gag have also been shown to contribute to optimal encapsidation of HIV-1 gRNA (Luban and Goff 1994; McBride et al. 1997; Helga-Maria et al. 1999; Clever et al. 2002; Russell et al. 2002; for review, see D'Souza and Summers 2005), although conformational perturbations could be at the origin of these effects (Ooms et al. 2004). Indeed, deletion of the DIS (Clever and Parslow 1997; Russell et al. 2003) more profoundly affects genomic RNA packaging than deletion of SL3 (Houzet et al. 2007). Besides these cis-acting elements, gRNA packaging requires the nucleocapsid (NC) domain of Gag. NC proteins have been shown to bind to the $5^{\prime}$ leader region of HIV-1 RNA (SL1-SL4, Fig. 1A) in vitro and to promote RNA dimerization via the DIS (Muriaux et al. 1996; Takahashi et al. 2001), and mutations in NC strongly affect viral infectivity by inhibiting gRNA packaging (Berkowitz et al. 1995; Zhang and Barklis 1995; D’Souza and Summers 2005).

More than 30 different mRNA species are produced by alternative splicing of the HIV-1 primary transcript due to the presence of $5 \mathrm{SD}$ and 9 splice acceptor (SA) sites (Purcell and Martin 1993; Cochrane et al. 2006; Stoltzfus and Madsen 2006). Following transcription, the $9.2 \mathrm{~kb}$ RNA gives rise to multispliced $2 \mathrm{~kb}$ RNAs encoding the regulatory proteins $\mathrm{Tat}, \mathrm{Rev}$, and the auxiliary protein Nef (Fig. 1B). Once a threshold level of Rev is reached 
(Pomerantz et al. 1992), splicing activity is reduced, and singly spliced $4 \mathrm{~kb}$ RNA species encoding Env, Vpu, Vif, and Vpr proteins are produced (Fig. 1B; Pomerantz et al. 1992). While singly and multispliced mRNAs are largely excluded from viral particles, significant amounts of cellular and viral mRNAs can be encapsidated when cis or trans packaging signals are disrupted (Clever and Parslow 1997; Muriaux et al. 2001; Russell et al. 2003; Onafuwa-Nuga et al. 2006; Roy et al. 2006). Noticeably, all HIV-1 spliced mRNAs contain most of the extended packaging signal found in the 5' UTR, including TAR, PBS, and DIS (Fig. 1). Moreover, considering that RNA dimerization is a prerequisite for RNA packaging, spliced viral RNAs containing the DIS may be able not only to homodimerize but also to form heterodimers with gRNA and compete with gRNA homodimers for packaging. However, the packaging efficiency of spliced HIV-1 RNAs is 50-fold lower than the packaging efficiency of gRNA (Houzet et al. 2007), suggesting that spliced RNAs are unable to form homo- and heterodimers or that these dimers are not packaged (Liang et al. 2004).

To shed light on this issue, we investigated the in vitro dimerization properties of spliced HIV-1 RNAs. We found that singly spliced (env, vpr) and multispliced (tat, rev, and nef) RNA fragments are able to homodimerize and to form heterodimers with genomic RNA in vitro. Chemical probing experiments and an antisense oligonucleotide directed against the DIS region indicated that the DIS is structurally functional and that dimerization of spliced HIV-1 RNA occurs through a loop-loop interaction. In addition, we showed by coupling in vitro transcription and dimerization assays of short (300 nt) and long (4000 nt) RNA fragments that RNA dimerization can take place during transcription and could affect the fate of HIV-1 RNAs by modulating splicing, transport, or/and localization.

\section{RESULTS}

\section{Spliced HIV-1 RNA fragments efficiently dimerize in vitro though a loop-loop interaction}

Many studies previously showed that fragments of HIV-1 gRNA are able to dimerize in vitro through an RNA-RNA interaction involving the self-complementary sequence in the DIS loop (Laughrea and Jetté 1994; Paillart et al. 1994; Skripkin et al. 1994). While the DIS sequence is localized upstream of the SD site and is therefore present in all spliced mRNAs of HIV-1 (Fig. 1), dimerization of HIV-1 mRNAs has never been specifically analyzed. The close proximity of the DIS and the 5' SD site (SD1, Fig. 1) could locally influence the secondary and tertiary RNA structures and make these mRNA noncompetent for dimerization; this would explain, at least partially, their exclusion from the viral particles. Representative RNA fragments corre- sponding to the first $615 \mathrm{nt}$ of singly spliced (vpr and env) and multispliced (rev, tat, and nef) HIV-1 mRNAs (Fig. 1B, isolate NL4.3) have been synthesized and tested for their ability to dimerize in vitro in conditions previously described to favor either monomer or dimer formation (Fig. 2A; Marquet et al. 1991; Paillart et al. 1994). As expected from the presence of the DIS sequence, all mRNA fragments were able to dimerize, as indicated by the appearance of a retarded band on agarose gel when the samples were incubated in "dimer buffer." For most mRNA fragments, the dimer yields were similar to that of the 1-615 gRNA fragment (between $51 \%$ and $68 \%$ of dimer) (Fig. 2A). The only exception was RNA vpr $1 / 3$, which was almost exclusively dimeric (dimer yield of $92 \%$ ).

To test whether dimerization of the HIV-1 spliced mRNAs involved formation of a kissing-loop complex via the DIS, as previously demonstrated for HIV-1 gRNA (Laughrea and Jetté 1994; Paillart et al. 1994; Skripkin et al. 1994; Muriaux et al. 1995), we co-incubated each mRNA fragment with a fivefold excess of an antisense oligodeoxyribonucleotide (dAS35) directed against the DIS stemloop (Fig. 2B; Skripkin et al. 1996; Lodmell et al. 1998). The dimerization of all mRNA fragments, including RNA vpr1/ 3, was inhibited by the presence of dAS35 (Fig. 2B, lanes marked + ). Similar results were obtained when dAS35 was added after dimer formation (data not shown). Taken together, these results indicate that (1) spliced HIV-1 mRNAs, like gRNA, are able to dimerize in vitro via the loop of the DIS; (2) this RNA-RNA interaction is dynamic; and (3) sequences downstream the SD do not contain redundant dimerization signals.
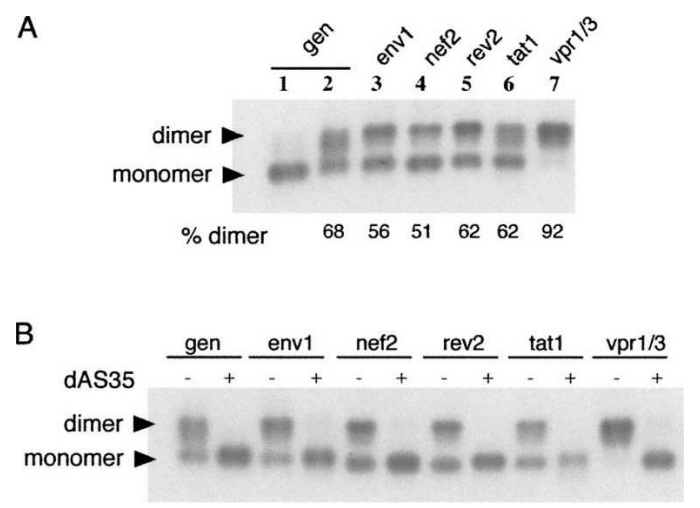

FIGURE 2. In vitro dimerization of spliced HIV-1 RNAs. (A) RNA fragments were incubated in dimer buffer (high-salt conditions) and run on a native $0.8 \%$ agarose gel (TB $0.5 \times, 0.1 \mathrm{mM} \mathrm{MgCl}_{2}$ ). Control monomeric RNA (low-salt conditions) is shown only for the genomic RNA fragment (lane 1). Monomers and dimers are indicated, as well as the percentage of dimer formed for each RNA. (B) Inhibition of RNA dimerization. RNAs were incubated in high-salt conditions in the absence $(-)$ or presence $(+)$ of an antisense oligodeoxynucleotide (dAS35) complementary to the DIS stem-loop. 


\section{Regions downstream of the DIS are important for the dimer stability}

In HIV-1 genomic RNA, sequences located in the first 300 nt downstream from SD1 have been shown to be involved in stabilization of the RNA dimer (Marquet et al. 1994; Paillart et al. 1994; Laughrea and Jette 1996). The molecular mechanism of this stabilization is still unknown, and the sequences involved remain to be precisely mapped. To test whether the different sequences located $3^{\prime}$ of SD1 of the different HIV-1 spliced mRNAs modulate the stability of the RNA dimers, we determined the thermal stability of the dimers by incubating these RNAs at $37^{\circ} \mathrm{C}$, then gradually increasing the temperature up to $62^{\circ} \mathrm{C}$. Samples were analyzed by agarose gel electrophoresis, and conversion of the dimer band into monomer with increasing temperature was quantified and compared with gRNA (see Materials and Methods; Fig. 3). We observed that spliced RNA fragments could be classified into two categories, according to the thermal stability $\left(\mathrm{T}_{\mathrm{m}}\right)$ of their dimers: the first one contains RNAs tat, rev, nef, and env, which had a $\mathrm{T}_{\mathrm{m}}$ $\left(47^{\circ} \mathrm{C}-52^{\circ} \mathrm{C}\right)$ slightly lower than the one determined for the gRNA fragment $\left(55^{\circ} \mathrm{C}\right)$; the second one corresponds to RNA vpr, which formed an extremely thermostable dimer $\left(\mathrm{T}_{\mathrm{m}}>65^{\circ} \mathrm{C}\right)$ (Fig. 3B). Thus, sequences downstream of the SD1 site of spliced mRNA fragments influenced the stability of RNA dimers either negatively, for tat, rev, nef, and env RNA species, or positively, for the vpr RNA, suggesting that, as for gRNA, stability of the mRNA dimers is not only due to Watson-Crick base-pairing of the selfcomplementary sequence of the DIS.

\section{The RNA structure upstream of SD1 is similar in HIV-1 spliced and genomic RNAs}

While all spliced RNAs dimerized through the DIS motif, their different dimer yield and thermal stability suggested that conformational differences could affect the presentation of the DIS sequence. In order to determine structural changes between HIV-1 spliced and gRNA fragments, we probed the region upstream of SD1, including the DIS, with dimethyl sulfate (DMS), a chemical probe that selectively methylates Watson-Crick positions of unpaired adenines (A-N1) and cytosines $(\mathrm{C}-\mathrm{N} 3)$ and $\mathrm{N}-7$ positions of guanines (G-N7) (Brunel and Romby 2000). We monitored the reactivity profile of adenines and cytosines in the spliced RNAs and compared it with the one obtained for gRNA (Fig. 4). In general, the global reactivity pattern of the DIS sequence was similar for all RNAs: (1) the three purines, A255, A256, and A263, surrounding the selfcomplementary sequence in the loop were reactive, and thus unpaired, with A263 showing a hyper-reactivity toward DMS, as previously observed for HIV-1 gRNA (Fig. 4A; Paillart et al. 1997); (2) cytidines of the selfcomplementary loop sequence (257GCGCGC262) were
A
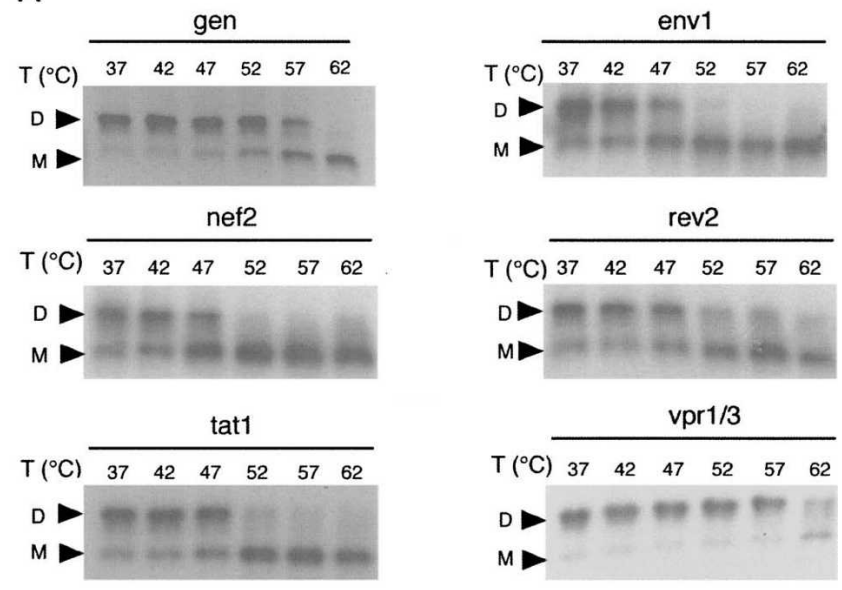

vpr1/3

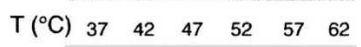

D

B

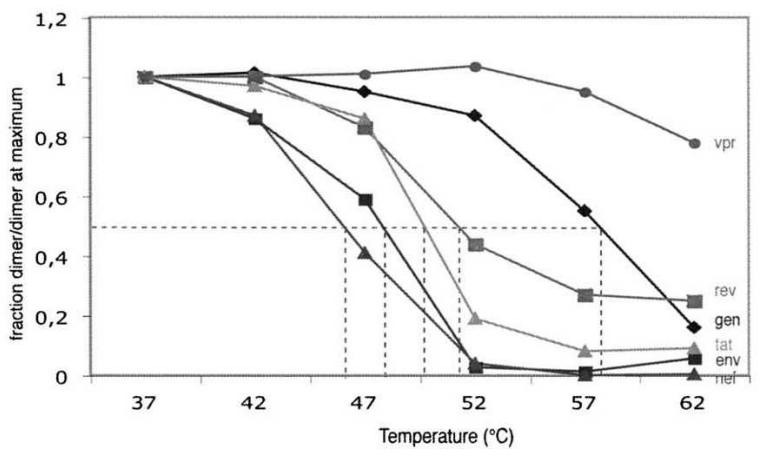

FIGURE 3. Thermal stability of genomic and spliced 1-615 RNA dimers. (A) After RNA dimerization in high-salt buffer at $37^{\circ} \mathrm{C}$, samples were incubated at different temperatures ranging from $37^{\circ} \mathrm{C}$ to $62^{\circ} \mathrm{C}$ and loaded on $0.8 \%$ native agarose gels. Gels were fixed, dried, and autoradiographed. (B) Thermal stability curves. Dimer yields were normalized relative to the dimer yield at $37^{\circ} \mathrm{C}$ and plotted as a function of temperature.

protected from methylation, as expected from the implication of the DIS in RNA dimerization; and (3) adenine A271 in the internal loop of the DIS stem was also modified by the probe (Fig. 4A,B). Moreover, we observed that the conformation of the region upstream of the DIS was similar in all mRNAs and that binding of oligodeoxynucleotide dAS35 did not affect this region, whereas the DIS loop was completely protected by dAS35 binding (data not shown). However, as previously observed (Paillart et al. 2004a), nucleotides A268 and A269 were slightly reactive toward DMS in gRNA as well as in vpr mRNA (Fig. 4). Surprisingly, these nucleotides appeared more reactive in all other spliced mRNAs (env, nef, rev, and tat), suggesting that the DIS upper stem is weaker in these mRNAs. Altogether, these results support the presence of the DIS stem-loop in all spliced RNAs and in the gRNA, with the loop sequence of one RNA interacting with the loop of another RNA molecule, in agreement with our in vitro RNA dimerization assays. In addition, our probing data suggest that all RNAs 
A

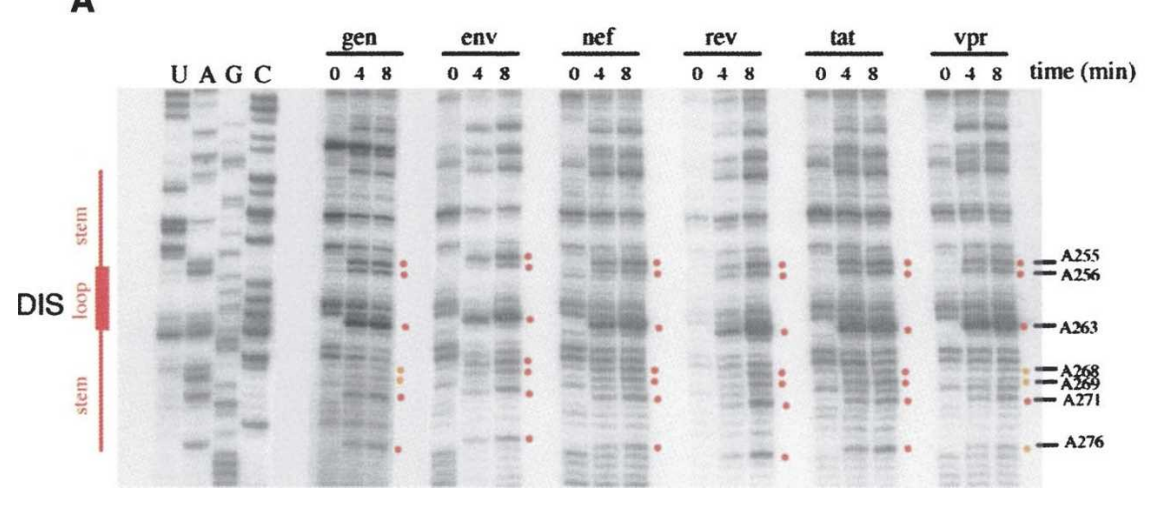

B

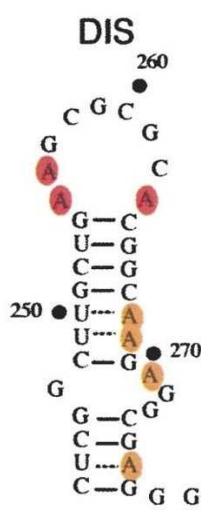

FIGURE 4. Dimethylsulfate (DMS) probing of the Dimerization Initiation Site (DIS) region of spliced and genomic HIV-1 RNAs. (A) RNAs were modified with DMS, and positions of methylated bases were revealed by primer extension before loading on an $8 \%$ polyacrylamide denaturing gel. Lanes U,A,C,G correspond to the sequence of gRNA. DIS and SD positions are indicated. Red dots represent the three purines flanking the self-complementary sequence of the DIS self-complementary sequence. (B) Secondary structure of the DIS. Nucleotides modified by DMS are indicated in red (highly reactive) or in orange (moderately reactive).

were structurally homogenous, in agreement with the electrophoresis analysis, which revealed that all monomeric species migrated as a single band (Fig. 2). Thus, our data strongly suggest that the dimerization yield and the thermal stability of the RNA dimers were not affected by an "RNA switch," such as the LDI-BMH equilibrium proposed for HIV-1 gRNA (Huthoff and Berkhout 2001; Berkhout et al. 2002).

\section{Heterodimer formation between spliced and unspliced RNA fragments is structurally constrained}

The self-complementary loop sequence of the DIS is required for dimerization of HIV-1 spliced and unspliced RNAs, and both classes of RNAs form the well-studied kissing-loop complex (Paillart et al. 2004b; Russell et al. 2004; D'Souza and Summers 2005). Thus, it is theoretically possible to form heterodimers between spliced and unspliced (genomic) RNAs by interaction of their DIS. Such heterodimers might favor packaging of spliced viral RNAs. To address this hypothesis, we first repeated the in vitro dimerization procedure by co-incubating each $615 \mathrm{nt}$ long spliced RNA fragment with an RNA fragment corresponding to the first $1402 \mathrm{nt}$ of HIV-1 gRNA (see Materials and Methods) and loading samples on a native agarose gel (Fig. 5A). Using RNA fragments of different lengths allowed us to distinguish homodimers from heterodimers. As seen in Figure 5A, a third retarded band was readily detected when unlabeled RNA 1-1402 was added to radiolabeled spliced or unspliced $615 \mathrm{nt}$ long RNA (Fig. 5A, lanes marked "+"). This band migrated between the positions of the dimeric forms of 1-615 RNA and 1-1402 RNA (Fig. 5A, lanes 1,2 ) and corresponded to a RNA heterodimer.

This experiment showed that it is possible to form heterodimers between spliced and unspliced HIV-1 RNA in vitro when these RNAs are heat-denaturated together in water, then co-incubated in dimerization buffer (see Materials and Methods). In order to avoid any possible artifact linked to the RNA denaturation/renaturation process, we next performed homo- and heterodimerization assays during in vitro run-off transcription from two templates generating the appropriate RNA fragments (Fig. 5B). As previously observed for MLV genomic RNA fragments (Kharytonchyk et al. 2005; Flynn and Telesnitsky 2006; Rasmussen and Pedersen 2006), spliced and unspliced HIV-1 RNAs were able to homodimerize during in vitro transcription (Fig. 5B, lanes $1,2,5,8,11,14,17$ ) with a yield similar to what was observed after RNA denaturation (Fig. 5A). In the same way, heterodimers between $615 \mathrm{nt}$ long spliced or unspliced RNA fragments and the 1-1402 gRNA fragment could easily be formed when templates were mixed together before starting in vitro transcription (Fig. 5B, lanes 3,6,9,12,15,18, red asterisks). In sharp contrast, when each RNA was generated in separate in vitro transcription reactions and subsequently incubated together for another $30 \mathrm{~min}$ at $37^{\circ} \mathrm{C}$, heterodimers could hardly be visualized on an agarose gel (Fig. 5B, lanes $4,7,10,13,16,19)$. The only exception was RNA rev2, which heterodimerized somewhat more efficiently (Fig. 5B, cf. lanes 12 and 13, gray asterisks). These results indicate that dimerization is more efficient during transcription than once RNA synthesis is completed, suggesting that accessibility of the DIS sequence is crucial for heterodimer formation, and for RNA dimerization in general. Alternatively, we cannot discard the possibility that the homodimers that are formed when the RNAs are incubated separately prevent subsequent formation of heterodimers when the RNAs are mixed together. However, this would imply that the RNA dimers are less dynamic than previously suggested (Paillart et al. 1996b). 

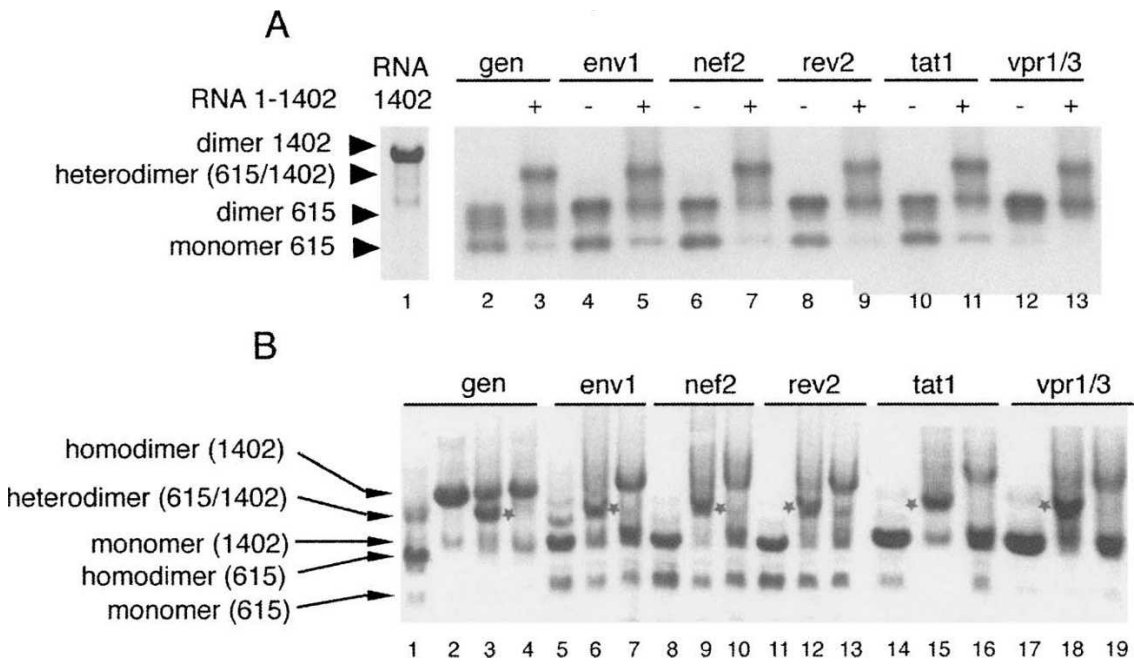

FIGURE 5. Heterodimerization of genomic and spliced HIV-1 RNAs. (A) Heterodimerization with purified RNA fragments. Radiolabeled 1-615 RNAs were incubated in high-salt buffer in the absence (-) or in presence (+) of genomic RNA fragment 1-1402. (Lane 1) The 1-1402 RNA dimer visualized by UV. Positions of monomer, homodimer, and heterodimer species are indicated. $(B)$ Heterodimer formation during in vitro transcription. DNA templates generating 1-615 spliced or genomic RNA fragments were submitted to transcription either alone (lanes $1,2,5,8,11,14,17$ ) or with template generating genomic RNA fragment 1-1402 (lanes $3,6,9,12,15,18)$. RNAs $1-615$ and $1-1402$ were also transcribed separately before being mixed together for another $30-\mathrm{min}$ incubation at $37^{\circ} \mathrm{C}$ (lanes 4,7,10,13,16,19). Samples were analyzed on a native ethidium-bromide-containing agarose gel. (Gray star) The 1-615/1-1402 heterodimer, (lane 1) genomic RNA 1-615, (lane 2) genomic RNA 1-1402. The names of each RNA are indicated at the top of the gel. (gen) gRNA.

\section{Dimerization of long RNA fragments is favored during in vitro transcription}

In vitro dimerization of retroviral RNA was usually restricted to RNA fragments of $<1500 \mathrm{nt}$, and the dimerization yield was usually found to sharply decrease as the size of the RNA fragments increased (Marquet et al. 1991, 1994; Flynn and Telesnitsky 2006). Inhibition of in vitro RNA dimerization with increasing RNA size might be due either to increasing electrostatic repulsion between the two RNA molecules or/and to reduced accessibility of the DIS in larger RNA fragments. In order to distinguish between these two possibilities, we analyzed dimerization of very long fragments of subtype A and subtype B HIV-1 gRNA during in vitro transcription (Fig. 6). RNA fragments up to $>4500 \mathrm{nt}$ efficiently dimerized during in vitro transcription (Fig. 6), whereas the longer RNA fragments only marginally dimerized when dimer formation was initiated after heat denaturation of fully synthesized RNAs (data not shown; Flynn and Telesnitsky 2006).

\section{DISCUSSION}

HIV-1 RNA packaging is a highly specific process that leads to the selection of a dimeric genomic RNA amongst a multitude of cellular and viral mRNAs. However, it is paradoxical that HIV-1 spliced RNAs, which contain most of the signals required for gRNA packaging (TAR, polyA, DIS), are not abundantly encapsidated into viral particles. Several hypotheses can be raised to explain this paradox: (1) HIV-1 spliced RNA could be compartmentalized in the cytoplasm, away from the assembly complex; (2) the conformation of the packaging motifs may not be suitable to direct efficient encapsidation of spliced mRNAs; or (3) mRNAs are not competent for dimerization, a prerequisite for RNA packaging (Paillart et al. 2004a; Moore et al. 2007). In this report, we analyzed for the first time the in vitro dimerization of several HIV-1 RNA fragments corresponding to the $5^{\prime}$ end of spliced mRNAs encoding the viral proteins Env, Nef, Vpu, Tat, Rev, and Nef.

Our results show that the structure of the DIS domain is conserved in all spliced RNAs and that these RNAs are able to dimerize in vitro through a kissing-loop mechanism involving the DIS loop (Fig. 2). Indeed, an oligonucleotide complementary to the DIS stem-loop completely abolished dimerization of spliced RNAs (Fig. 2B), suggesting that, as for gRNA (Skripkin et al. 1996), dimerization of spliced RNA is in dynamic equilibrium and governed by reversible loop-loop interactions. Moreover, this inhibition indicates that no sequence downstream of the SD site (region 288-615 in our study) can complement the loss of the DIS function, reinforcing the idea that the main dimerization signal is not redundant. In addition, we observed a rather good correlation between the dimerization yield (Fig. 2) and the in vitro thermal stability of spliced mRNA (Fig. 4). Spliced RNAs can be divided into two categories: (1) nef, env, tat, and rev with a dimerization yield $(51 \%-62 \%)$ and a thermal stability $\left(\mathrm{T}_{\mathrm{m}}\right.$, ranging from $46^{\circ} \mathrm{C}$ to $\left.52^{\circ} \mathrm{C}\right)$ lower than that of the gRNA $\left(68 \%\right.$ and $\mathrm{T}_{\mathrm{m}}$ $\sim 57^{\circ} \mathrm{C}$ ); and (2) vpr, which formed $>90 \%$ of dimer with a surprisingly high thermal stability $\left(>70^{\circ} \mathrm{C}\right)$ (Fig. 4). These results suggest that the sequence or the conformation of spliced RNAs must be important for the stability of RNA dimers. However, our chemical probing indicates that the structure of the DIS stem-loop and of the $5^{\prime}$ region is not significantly different in all HIV-1 RNAs, suggesting that the deletion of the intronic sequence during splicing does not significantly alter the conformation of neighboring sequences. Thus, the mechanism by which the $v p r$ sequences stabilize the RNA dimer is unclear. It is, however, important to notice that the downstream sequence that is removed upon splicing has either a negative influence in the wild-type RNA stability or a positive influence imposed by the unique 


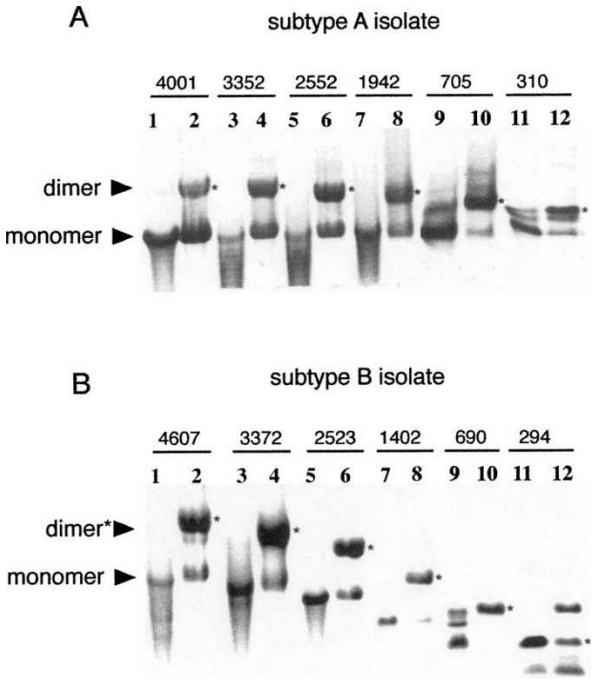

FIGURE 6. In vitro dimerization of long subtype A $(A)$ and subtype B (B) RNA fragments during transcription. Samples were analyzed on native agarose ethidium-bromide-containing gels directly after in vitro run-off transcription. (Black stars) Dimers. The size of each RNA fragment is indicated at the top of the gels. (Odd lanes) RNA fragments that have been denatured $2 \mathrm{~min}$ at $80^{\circ} \mathrm{C}$ before loading, (even lanes) not heat-treated. RNA fragments are staggered in panel $B$ (compared with panel $A$ ) due to direct loading of samples after transcription. In this case, RNAs migrated according to their length. In panel $A$, RNA samples have been loaded at different time points to place the RNA bands at the same position in the gel.

downstream sequence in the vpr mRNA. Structure prediction using the Mfold program did not allow us to pinpoint a specific region involved in the stabilization capacity of this mRNA (data not shown). However, the weaker DIS stem observed for env, nef, rev, and tat RNA fragments can potentially be involved in the reduced thermal stability determined for these RNAs (Fig. 3). Similarly, in vitro studies showed that sequences in gag stabilize the dimer of gRNA (Paillart et al. 1994; Laughrea and Jette 1997), whereas analysis of mutant viruses indicated that regions in pol have a destabilizing effect (Shehu-Xhilaga et al. 2001, 2002). The precise identification of the sequences and mechanisms involved in the modulation of the RNA dimer stability remain to be determined. The loss or the formation of long distance intra- or intermolecular interactions between regions on both sides of the SD site or with the DIS stem-loop by itself could thus explain the thermal stability differences between spliced RNAs. Nevertheless, whatever these undefined sequences or their conformations, they do not affect the dimerization process, as all HIV-1 spliced RNA fragments dimerized efficiently. This study has to be compared with the one by Lanchy et al. (2004), which showed that removing intronic sequence from HIV-2 RNA (as in tat, rev, and nef mRNAs) only locally affected the RNA conformation of RNAs. As the main packaging signal is located upstream of the SD site in HIV-2, these investigators suggested that the interaction between elements upstream and downstream of the SD site may help to select gRNA preferentially over spliced RNAs during packaging.

If we extrapolate these results to the in vivo context, they indicate that spliced RNA could dimerize in the cells and thus that spliced and gRNAs could compete for dimer formation and packaging, as recently proposed (Houzet et al. 2007). These results prompted us to analyze the capacity of spliced RNA to form heterodimers with gRNA. Indeed, we observed that all spliced RNAs efficiently form heterodimers in vitro. As one molecule of these heterodimers possesses the complete packaging signal, one could expect efficient packaging of these heterodimers. However, experiments showed that spliced RNAs are packaged with low efficiency (Liang et al. 2004; Houzet et al. 2007). In addition, we recently showed that while deletion of SL1 strongly affects packaging of gRNA, it has no direct effect on the packaging of spliced HIV-1 RNA (Houzet et al. 2007). Thus, either heterodimers are not formed in cells, and the mechanism preventing heterodimerization remains to be elucidated, or they are not recognized by the packaging machinery, and understanding how this machinery can discriminate between heterodimers and dimers of gRNA would certainly shed a new light on the role of RNA dimerization in RNA packaging.

Finally, we showed that heterodimerization and dimerization of long gRNA fragments is more efficient during in vitro transcription than after complete synthesis of these RNAs (Figs. 5, 6). Thus, in infected cells, RNA dimerization could occur during transcription of the integrated provirus, in agreement with previous in vitro and ex vivo studies on Moloney murine leukemia viruses (Kharytonchyk et al. 2005; Flynn and Telesnitsky 2006; Rasmussen and Pedersen 2006) showing that gRNAs synthesized from separated locations do not randomly associate. As splicing of HIV-1 RNA is inefficient, as compared with cellular mRNA, RNA dimerization might precede and modulate splicing. For instance, splicing of the two RNA molecules within a dimer may be coupled, even though deletion of the DIS motif in gRNA does not globally affect the amount of spliced HIV-1 RNA (Houzet et al. 2007).

Whether RNA dimerization or/and heterodimerization can occur in the nucleus during transcription is still unclear. Work is in progress in our laboratory to test this possibility. Indeed, nuclear dimerization of HIV-1 RNAs could have important functional consequences for HIV-1 RNA metabolism, such as nuclear export, trafficking, and packaging (Basyuk et al. 2005; Poole et al. 2005; Smagulova et al. 2005; Levesque et al. 2006; Swanson and Malim 2006).

\section{MATERIALS AND METHODS}

\section{Plasmid construction and RNA synthesis}

Plasmid pNL4.3-615 expresses the first 615 nt of the HIV-1 NL4.3 isolate under the control of the T7 RNA polymerase promoter 
(Lodmell et al. 1998). Plasmids pSP5 containing the complete cDNAs expressing the Env/Vpu, Nef, Rev, Tat, and Vpr mRNAs of HIV-1 NL4.3 (Purcell and Martin 1993) were PCR amplified (4 min at $94^{\circ} \mathrm{C}$; and 30 cycles of $1 \mathrm{~min}$ at $94^{\circ} \mathrm{C}, 1 \mathrm{~min}$ at $52^{\circ} \mathrm{C}$, and $1 \mathrm{~min}$ at $72^{\circ} \mathrm{C}$ ) and inserted into PCR 2.1 -TOPO vectors (Invitrogen). The resulting plasmids pT-env/vpu, pT-nef, pT-rev, pTtat, and pT-vpr1/3 contain the first $615 \mathrm{nt}$ of HIV-1 mRNAs downstream from the T7 promoter.

Plasmids pNL4.3-615 and pTs were linearized with PvuII and EcoRI, respectively, prior to in vitro transcription to generate RNAs encompassing nucleotides 1-615 of HIV-1 gRNA and mRNAs (Fig. 1). After run-off transcription, RNAs were purified by FPLC as previously described (Henriet et al. 2005). Internal labeling of RNA was achieved by addition of $\left[\alpha-{ }^{32} \mathrm{P}\right]$ ATP (Amersham) during transcription (Paillart et al. 1996b).

Plasmids pmCG67 (Marquet et al. 1991) and pDR4607 contain the $5^{\prime}$ half of the MAL and NL4.3 HIV-1 genome, respectively, under the control of the T7 promoter. Plasmid pmCG67 was digested with RsaI, PvuII, AvaII, BstXI, EcoRV, BamHI, or SalI to produce RNA 1-311 (containing the first 311 nt of RNA genome), RNA 1-705, RNA 1-1333, RNA 1-1942, RNA 1-2552, RNA 1-3352, and RNA 1-4001, respectively. Likewise, pDR4607 was digested with RsaI, PvuII, MspI, EcoRV, KpnI, or SmaI to produce RNA 1-294, RNA 1-690, RNA 1-1402, RNA 1-2523, RNA 1-3372, and RNA 1-4607, respectively.

\section{In vitro dimerization of HIV-1 RNAs}

In a typical experiment, $400 \mathrm{nM}$ of unlabeled RNA fragments were diluted in $8 \mu \mathrm{L}$ of Milli-Q (Millipore) water with the corresponding labeled RNA (5000 cpm, 3-5 nM). Samples were denatured for $2 \mathrm{~min}$ at $90^{\circ} \mathrm{C}$ and snap-cooled for $2 \mathrm{~min}$ on ice. Dimerization was initiated by addition of fivefold concentrated dimer buffer (final concentration: $50 \mathrm{mM}$ sodium cacodylate, $\mathrm{pH}$ 7.5, $300 \mathrm{mM}$ $\mathrm{KCl}$, and $5 \mathrm{mM} \mathrm{MgCl}_{2}$ ), and the samples were incubated for $30 \mathrm{~min}$ at $37^{\circ} \mathrm{C}$. In parallel, samples were incubated with fivefold monomer buffer (final concentration: $50 \mathrm{mM}$ sodium cacodylate, $\mathrm{pH} 7.5,40 \mathrm{mM} \mathrm{KCl}$, and $0.1 \mathrm{mM} \mathrm{MgCl}_{2}$ ) to induce folding of monomeric RNA. Samples were loaded on a $0.8 \%$ agarose gel containing ethidium bromide in TBM buffer $(0.5 \times$ Tris-Borate, $0.1 \mathrm{mM} \mathrm{MgCl}_{2}$ ) and run at $4^{\circ} \mathrm{C}$. Gels were fixed in $10 \%$ trichloroacetic acid for $10 \mathrm{~min}$ and dried for $1 \mathrm{~h}$ under vacuum at room temperature. Radioactive bands corresponding to monomeric and dimeric species were visualized and quantified using a FLA 5000 (Fuji).

To determine the thermal stability of the dimer species, samples were incubated for $30 \mathrm{~min}$ at $37^{\circ} \mathrm{C}$ as described above, and the temperature was gradually increased by $5^{\circ} \mathrm{C}$ steps up to $62^{\circ} \mathrm{C}$. After a 10 -min incubation at the appropriate temperature, an aliquot was loaded on a $0.8 \%$ agarose gel after addition of loading buffer. The melting temperature $\left(\mathrm{T}_{\mathrm{m}}\right)$ was defined as the temperature at which the amount of dimeric RNA was reduced by $50 \%$, as compared with its value at $37^{\circ} \mathrm{C}$.

For heterodimerization experiments, $400 \mathrm{nM}$ of RNA corresponding to the first $1402 \mathrm{nt}$ of HIV-1 NL4.3 gRNA were added to an equimolar concentration of 1-615 RNA containing $5000 \mathrm{cpm}$ of the corresponding labeled RNA. Heterodimerization assays were performed as described above for homodimerization.

\section{Inhibition of RNA dimerization by an antisense oligonucleotide}

RNA dimerization was performed as described above, then 50 pmol of unlabeled antisense oligodeoxynucleotide (dAS35) complementary to positions 243-277 (DIS stem-loop) of the HIV-1 NL4.3 gRNA were added, and incubation was continued for 15 min. Alternatively, the antisense oligodeoxynucleotide was added prior to the thermal denaturation step. Inhibition of RNA dimerization was visualized on agarose gel as above.

\section{Dimerization of HIV-1 RNAs during in vitro transcription}

One microgram of DNA templates digested by PvuII (pNL4.3615) or EcoRI (pTs) was transcribed in vitro as described above in a final volume of $20 \mu \mathrm{L}$. After incubation for $2 \mathrm{~h}$ at $37^{\circ} \mathrm{C}$, samples were phenol/chloroform extracted and directly loaded onto a $0.8 \%$ agarose gel containing ethidium bromide. Gels were run in TBM buffer at $4^{\circ} \mathrm{C}$. Heterodimer formation during in vitro transcription was performed similarly by mixing two different restricted DNA templates. Monomer, dimer, and heterodimer species were visualized by UV and analyzed with a Chemidoc (Bio-Rad) and the QuantityOne software. Alternatively, both templates were in vitro transcribed separately, then mixed together and incubated for $20 \mathrm{~min}$ at $37^{\circ} \mathrm{C}$ to allow heterodimer formation.

\section{Chemical probing of the DIS region of genomic and spliced RNAs}

Chemical probing with dimethylsulfate (DMS) was used for mapping the secondary structure of genomic or spliced RNA fragments. RNAs 1-615 (400 nM) were submitted to the dimerization procedure as described above, followed by a $10 \mathrm{~min}$ incubation at room temperature. Samples supplemented with $2 \mu \mathrm{g}$ of total Escherichia coli tRNA were subsequently modified for 0,4 , or $8 \mathrm{~min}$ at $37^{\circ} \mathrm{C}$ with $0.8 \mu \mathrm{L}$ of DMS diluted 20 -fold with ethanol. Modification reactions were stopped by ethanol precipitation, and pellets were washed twice with $75 \%$ ethanol, vacuumdried, and resuspended in $7 \mu \mathrm{L}$ of water. DMS modifications were detected by primer extension with avian myeloblastosis reverse transcriptase as previously described (Paillart et al. 1997). Sequencing reactions were performed in parallel to identify positions of DMS modifications. After reverse transcription, samples were ethanol precipitated, resuspended in $8 \mu \mathrm{L}$ of formamide loading buffer, and loaded onto an $8 \%$ denaturing polyacrylamide gel. After migration, gels were dried and autoradiographied at $-80^{\circ} \mathrm{C}$.

\section{ACKNOWLEDGMENTS}

This work was supported by a grant from the Agence Nationale de Recherches sur le SIDA (ANRS). L.S. is supported by a fellowship from the French Ministry of Research and Technology (MENRT).

Received June 8, 2007; accepted August 28, 2007.

\section{REFERENCES}

Aldovini, A. and Young, R.A. 1990. Mutations of RNA and protein sequences involved in human immunodeficiency virus type 1 
packaging result in production of noninfectious virus. J. Virol. 64: 1920-1926.

Andersen, E.S., Jeeninga, R.E., Damgaard, C.K., Berkhout, B., and Kjems, J. 2003. Dimerization and template switching in the $5^{\prime}$ untranslated region between various subtypes of human immunodeficiency virus type 1. J. Virol. 77: 3020-3030.

Balakrishnan, M., Fay, P.J., and Bambara, R.A. 2001. The kissing hairpin sequence promotes recombination within the HIV-I 5' leader region. J. Biol. Chem. 276: 36482-36492.

Balakrishnan, M., Roques, B.P., Fay, P.J., and Bambara, R.A. 2003. Template dimerization promotes an acceptor invasion-induced transfer mechanism during human immunodeficiency virus type 1 minus-strand synthesis. J. Virol. 77: 4710-4721.

Basyuk, E., Boulon, S., Skou Pedersen, F., Bertrand, E., and Vestergaard Rasmussen, S. 2005. The packaging signal of MLV is an integrated module that mediates intracellular transport of genomic RNAs. J. Mol. Biol. 354: 330-339.

Bender, W. and Davidson, N. 1976. Mapping of poly(A) sequences in the electron microscope reveals unusual structure of type $\mathrm{C}$ oncornavirus RNA molecules. Cell 7: 595-607.

Berkhout, B. and van Wamel, J.L. 1996. Role of the DIS hairpin in replication of human immunodeficiency virus type 1. J. Virol. 70: 6723-6732.

Berkhout, B., Ooms, M., Beerens, N., Huthoff, H., Southern, E., and Verhoef, K. 2002. In vitro evidence that the untranslated leader of the HIV-1 genome is an RNA checkpoint that regulates multiple functions through conformational changes. J. Biol. Chem. 277: 19967-19975.

Berkowitz, R.D., Ohagen, A., Hoglund, S., and Goff, S.P. 1995. Retroviral nucleocapsid domains mediate the specific recognition of genomic viral RNAs by chimeric Gag polyproteins during RNA packaging in vivo. J. Virol. 69: 6445-6456.

Brunel, C. and Romby, P. 2000. Probing RNA structure and RNA-ligand complexes with chemical probes. Methods Enzymol. 318: 3-21.

Chin, M.P., Rhodes, T.D., Chen, J., Fu, W., and Hu, W.S. 2005. Identification of a major restriction in HIV-1 intersubtype recombination. Proc. Natl. Acad. Sci. 102: 9002-9007.

Clavel, F. and Orenstein, J.M. 1990. A mutant of HIV-1 with reduced RNA packaging and abnormal particle morphology. J. Virol. 64: 5230-5234.

Clever, J.L. and Parslow, T.G. 1997. Mutant human immunodeficiency virus type 1 genomes with defects in RNA dimerization or encapsidation. J. Virol. 71: 3407-3414.

Clever, J.L., Wong, M.L., and Parslow, T.G. 1996. Requirements for kissing-loop-mediated dimerization of human immunodeficiency virus RNA. J. Virol. 70: 5902-5908.

Clever, J.L., Mirandar Jr., D., and Parslow, T.G. 2002. RNA structure and packaging signals in the $5^{\prime}$ leader region of the human immunodeficiency virus type 1 genome. J. Virol. 76: 12381-12387.

Cochrane, A.W., McNally, M.T., and Mouland, A.J. 2006. The retrovirus RNA trafficking granule: From birth to maturity. Retrovirology 3: 18. doi: 10.1186/1742-4690-3-18.

D'Souza, V. and Summers, M.F. 2005. How retroviruses select their genomes. Nat. Rev. Microbiol. 3: 643-655.

Feng, Y.X., Moore, S.P., Garfinkel, D.J., and Rein, A. 2000. The genomic RNA in Tyl virus-like particles is dimeric. J. Virol. 74: 10819-10821.

Flynn, J.A. and Telesnitsky, A. 2006. Two distinct Moloney murine leukemia virus RNAs produced from a single locus dimerize at random. Virology 344: 391-400.

$\mathrm{Fu}, \mathrm{W}$. and Rein, A. 1993. Maturation of dimeric viral RNA of Moloney murine leukemia virus. J. Virol. 67: 5443-5449.

Fu, W., Gorelick, R.J., and Rein, A. 1994. Characterization of human immunodeficiency virus type 1 dimeric RNA from wild-type and protease-defective virions. J. Virol. 68: 5013-5018.

Haddrick, M., Lear, A.L., Cann, A.J., and Heaphy, S. 1996. Evidence that a kissing loop structure facilitates genomic RNA dimerisation in HIV-1. J. Mol. Biol. 259: 58-68.
Helga-Maria, C., Hammarskjold, M.L., and Rekosh, D. 1999. An intact TAR element and cytoplasmic localization are necessary for efficient packaging of human immunodeficiency virus type 1 genomic RNA. J. Virol. 73: 4127-4135.

Henriet, S., Richer, D., Bernacchi, S., Decroly, E., Vigne, R., Ehresmann, B., Ehresmann, C., Paillart, J.C., and Marquet, R. 2005. Cooperative and specific binding of Vif to the $5^{\prime}$ region of HIV-1 genomic RNA. J. Mol. Biol. 354: 55-72.

Houzet, L., Paillart, J.-C., Smagulova, F., Maurel, S., Morichaud, Z., Marquet, R., and Mougel, M. 2007. HIV controls the selective packaging of genomic, spliced viral and cellular RNAs into virions through different mechanisms. Nucleic Acids Res. 35: 2695-2704. doi: $10.1093 / \mathrm{nar} / \mathrm{gkm} 153$.

Huthoff, H. and Berkhout, B. 2001. Two alternating structures of the HIV-1 leader RNA. RNA 7: 143-157.

Kharytonchyk, S.A., Kireyeva, A.I., Osipovich, A.B., and Fomin, I.K. 2005. Evidence for preferential copackaging of Moloney murine leukemia virus genomic RNAs transcribed in the same chromosomal site. Retrovirology 2: 3.

Lanchy, J.M., Szafran, Q.N., and Lodmell, J.S. 2004. Splicing affects presentation of RNA dimerization signals in HIV-2 in vitro. Nucleic Acids Res. 32: 4585-4595. doi: 10.1093/nar/gkh800.

Laughrea, M. and Jetté, L. 1994. A 19-nucleotide sequence upstream of the $5^{\prime}$ major splice donor site is part of the dimerization domain of human immunodeficiency virus 1 genomic RNA. Biochemistry 33: $13464-13474$.

Laughrea, M. and Jette, L. 1996. HIV-1 genome dimerization: Formation kinetics and thermal stability of dimeric HIV-1Lai RNAs are not improved by the 1-232 and 296-790 regions flanking the kissing-loop domain. Biochemistry 35: 9366-9374.

Laughrea, M. and Jette, L. 1997. HIV-1 genome dimerization: Kissingloop hairpin dictates whether nucleotides downstream of the $5^{\prime}$ splice junction contribute to loose and tight dimerization of human immunodeficiency virus RNA. Biochemistry 36: 95019508.

Laughrea, M., Jette, L., Mak, J., Kleiman, L., Liang, C., and Wainberg, M. 1997. Mutations in the kissing-loop hairpin of human immunodeficiency virus type 1 reduce viral infectivity as well as genomic RNA packaging and dimerization. J. Virol. 71: 3397-3406.

Lever, A.M. 2000. HIV RNA packaging and lentivirus-based vectors. Adv. Pharmacol. 48: 1-28.

Lever, A., Gottlinger, H., Haseltine, W., and Sodroski, J. 1989. Identification of a sequence required for efficient packaging of HIV-1 RNA into virions. J. Virol. 63: 4085-4087.

Levesque, K., Halvorsen, M., Abrahamyan, L., Chatel-Chaix, L., Poupon, V., Gordon, H., DesGroseillers, L., Gatignol, A., and Mouland, A.J. 2006. Trafficking of HIV-1 RNA is mediated by heterogeneous nuclear ribonucleoprotein A2 expression and impacts on viral assembly. Traffic 7: 1177-1193.

Liang, C., Hu, J., Russell, R.S., Kameoka, M., and Wainberg, M.A. 2004. Spliced human immunodeficiency virus type 1 RNA is reverse transcribed into cDNA within infected cells. AIDS Res. Hum. Retroviruses 20: 203-211.

Lodmell, J.S., Paillart, J.C., Mignot, D., Ehresmann, B., Ehresmann, C., and Marquet, R. 1998. Oligonucleotide-mediated inhibition of genomic RNA dimerization of HIV-1 strains MAL and LAI: A comparative analysis. Antisense Nucleic Acid Drug Dev. 8: $517-529$.

Luban, J. and Goff, S.P. 1994. Mutational analysis of cis-acting packaging signal in human immunodeficiency virus type 1 RNA. J. Virol. 68: 3784-3793.

Marquet, R., Baudin, F., Gabus, C., Darlix, J.L., Mougel, M., Ehresmann, C., and Ehresmann, B. 1991. Dimerization of human immunodeficiency virus (type 1) RNA: Stimulation by cations and possible mechanism. Nucleic Acids Res. 19: 23492357.

Marquet, R., Paillart, J.-C., Skripkin, E., Ehresmann, C., and Ehresmann, B. 1994. Dimerization of human immunodeficiency 
virus type 1 RNA involves sequences located upstream of the splice donor site. Nucleic Acids Res. 22: 145-151.

McBride, M.S., Schwartz, M.D., and Panganiban, A.T. 1997. Efficient encapsidation of human immunodeficiency virus type 1 vectors and further characterization of cis elements required for encapsidation. J. Virol. 71: 4544-4554.

Moore, M.D., Fu, W., Nikolaitchik, O., Chen, J., Ptak, R.G., and $\mathrm{Hu}$, W.S. 2007. Dimer initiation signal of human immunodeficiency virus type I: Its role in partner selection during RNA copackaging and its effects on recombination. J. Virol. 81: 40024011.

Muriaux, D., Girard, P.M., Bonnet, M.B., and Paoletti, J. 1995. Dimerization of HIV-1Lai RNA at low ionic strength. An autocomplementary sequence in the $5^{\prime}$ leader region is evidenced by an antisense oligonucleotide. J. Biol. Chem. 270: 8209-8216.

Muriaux, D., De, R.H., Roques, B.P., and Paoletti, J. 1996. NCp7 activates HIV-1Lai RNA dimerization by converting a transient loop-loop complex into a stable dimer. J. Biol. Chem. 271: 3368633692.

Muriaux, D., Mirro, J., Harvin, D., and Rein, A. 2001. RNA is a structural element in retrovirus particles. Proc. Natl. Acad. Sci. 98: 5246-5251.

Murti, K.G., Bondurant, M., and Tereba, A. 1981. Secondary structural features in the 70S RNAs of Moloney murine leukemia and Rous sarcoma viruses as observed by electron microscopy. J. Virol. 37: 411-419.

Oertle, S. and Spahr, P.F. 1990. Role of the gag polyprotein precursor in packaging and maturation of Rous sarcoma virus genomic RNA. J. Virol. 64: 5757-5763.

Onafuwa-Nuga, A.A., Telesnitsky, A., and King, S.R. 2006. 7SL RNA, but not the 54-kd signal recognition particle protein, is an abundant component of both infectious HIV-1 and minimal virus-like particles. RNA 12: 542-546.

Ooms, M., Huthoff, H., Russell, R., Liang, C., and Berkhout, B. 2004. A riboswitch regulates RNA dimerization and packaging in human immunodeficiency virus type 1 virions. J. Virol. 78: 10814-10819.

Paillart, J.-C., Marquet, R., Skripkin, E., Ehresmann, B., and Ehresmann, C. 1994. Mutational analysis of the bipartite dimer linkage structure of human immunodeficiency virus type 1 genomic RNA. J. Biol. Chem. 269: 27486-27493.

Paillart, J.-C., Berthoux, L., Ottmann, M., Darlix, J.-L., Marquet, R., Ehresmann, B., and Ehresmann, C. 1996a. A dual role of the putative RNA dimerization initiation site of human immunodeficiency virus type 1 in genomic RNA packaging and proviral DNA synthesis. J. Virol. 70: 8348-8354.

Paillart, J.-C., Skripkin, E., Ehresmann, B., Ehresmann, C., and Marquet, R. 1996b. A loop-loop "kissing" complex is the essential part of the dimer linkage of genomic HIV-1 RNA. Proc. Natl. Acad. Sci. 93: 5572-5577.

Paillart, J.-C., Westhof, E., Ehresmann, C., Ehresmann, B., and Marquet, R. 1997. Non-canonical interactions in a kissing-loop complex: The dimerization initiation site of HIV-1 genomic RNA. J. Mol. Biol. 270: 36-49.

Paillart, J.C., Dettenhofer, M., Yu, X.F., Ehresmann, C., Ehresmann, B., and Marquet, R. 2004a. First snapshots of the HIV-1 RNA structure in infected cells and in virions. J. Biol. Chem. 279: 48397-48403.

Paillart, J.-C., Shehu-Xhilaga, M., Marquet, R., and Mak, J. 2004b. Dimerization of retroviral RNA genomes: An inseparable pair. Nat. Rev. Microbiol. 2: 461-472.

Pomerantz, R.J., Seshamma, T., and Trono, D. 1992. Efficient replication of human immunodeficiency virus type 1 requires a threshold level of Rev: Potential implications for latency. J. Virol. 66: 1809-1813.

Poole, E., Strappe, P., Mok, H.P., Hicks, R., and Lever, A.M. 2005. HIV-1 Gag-RNA interaction occurs at a perinuclear/centrosomal site; analysis by confocal microscopy and FRET. Traffic 6: 741-755.
Purcell, D.F. and Martin, M.A. 1993. Alternative splicing of human immunodeficiency virus type 1 mRNA modulates viral protein expression, replication, and infectivity. J. Virol. 67: 6365-6378.

Rasmussen, S.V. and Pedersen, F.S. 2006. Co-localization of gammaretroviral RNAs at their transcription site favors co-packaging. J. Gen. Virol. 87: 2279-2289.

Roy, B.B., Russell, R.S., Turner, D., and Liang, C. 2006. The T12I mutation within the SP1 region of Gag restricts packaging of spliced viral RNA into human immunodeficiency virus type 1 with mutated RNA packaging signals and mutated nucleocapsid sequence. Virology 344: 304-314.

Russell, R.S., Hu, J., Laughrea, M., Wainberg, M.A., and Liang, C. 2002. Deficient dimerization of human immunodeficiency virus type 1 RNA caused by mutations of the u5 RNA sequences. Virology 303: 152-163.

Russell, R.S., Roldan, A., Detorio, M., Hu, J., Wainberg, M.A., and Liang, C. 2003. Effects of a single amino acid substitution within the p2 region of human immunodeficiency virus type 1 on packaging of spliced viral RNA. J. Virol. 77: 12986-12995.

Russell, R.S., Liang, C., and Wainberg, M.A. 2004. Is HIV-1 RNA dimerization a prerequisite for packaging? Yes, no, probably? Retrovirology 1: 23. doi: 10.1186/1742-4690-1-23.

Shehu-Xhilaga, M., Crowe, S.M., and Mak, J. 2001. Maintenance of the Gag/Gag-Pol ratio is important for human immunodeficiency virus type 1 RNA dimerization and viral infectivity. J. Virol. 75: 1834-1841.

Shehu-Xhilaga, M., Hill, M., Marshall, J.A., Kappes, J., Crowe, S.M., and Mak, J. 2002. The conformation of the mature dimeric human immunodeficiency virus type 1 RNA genome requires packaging of pol protein. J. Virol. 76: 4331-4340.

Shen, N., Jette, L., Liang, C., Wainberg, M.A., and Laughrea, M. 2000. Impact of human immunodeficiency virus type 1 RNA dimerization on viral infectivity and of stem-loop B on RNA dimerization and reverse transcription and dissociation of dimerization from packaging. J. Virol. 74: 5729-5735.

Skripkin, E., Paillart, J.-C., Marquet, R., Ehresmann, B., and Ehresmann, C. 1994. Identification of the primary site of the human immunodeficiency virus type 1 RNA dimerization in vitro. Proc. Natl. Acad. Sci. 91: 4945-4949.

Skripkin, E., Paillart, J.-C., Marquet, R., Blumenfeld, M., Ehresmann, B., and Ehresmann, C. 1996. Mechanisms of inhibition of in vitro dimerization of HIV type I RNA by sense and antisense oligonucleotides. J. Biol. Chem. 271: 28812-28817.

Smagulova, F., Maurel, S., Morichaud, Z., Devaux, C., Mougel, M., and Houzet, L. 2005. The highly structured encapsidation signal of MuLV RNA is involved in the nuclear export of its unspliced RNA. J. Mol. Biol. 354: 1118-1128.

Stewart, L., Schatz, G., and Vogt, V.M. 1990. Properties of avian retrovirus particles defective in viral protease. J. Virol. 64: 50765092.

Stoltzfus, C.M. and Madsen, J.M. 2006. Role of viral splicing elements and cellular RNA binding proteins in regulation of HIV-1 alternative RNA splicing. Curr. HIV Res. 4: 43-55.

Swanson, C.M. and Malim, M.H. 2006. Retrovirus RNA trafficking: From chromatin to invasive genomes. Traffic 7: 1440-1450.

Takahashi, K.I., Baba, S., Chattopadhyay, P., Koyanagi, Y., Yamamoto, N., Takaku, H., and Kawai, G. 2000. Structural requirement for the two-step dimerization of human immunodeficiency virus type 1 genome. RNA 6: 96-102.

Takahashi, K., Baba, S., Koyanagi, Y., Yamamoto, N., Takaku, H., and Kawai, G. 2001. Two basic regions of NCp7 are sufficient for conformational conversion of HIV-1 dimerization initiation site from kissing-loop dimer to extended-duplex dimer. J. Biol. Chem. 276: 31274-31278

Zhang, Y. and Barklis, E. 1995. Nucleocapsid protein effects on the specificity of retrovirus RNA encapsidation. J. Virol. 69: 57165722. 

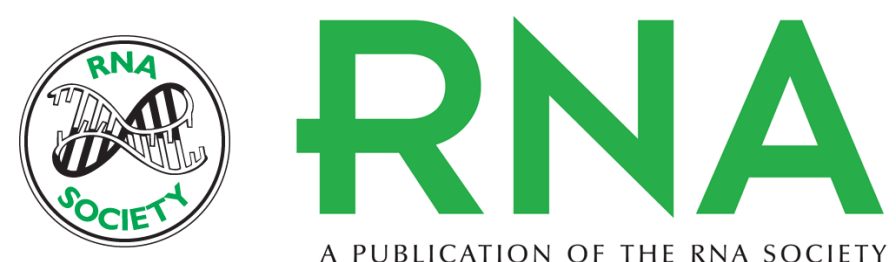

A PUBLICATION OF THE RNA SOCIETY

\section{In vitro dimerization of human immunodeficiency virus type 1 (HIV-1) spliced RNAs}

Lucile Sinck, Delphine Richer, Jane Howard, et al.

RNA 2007 13: 2141-2150

References This article cites 75 articles, 45 of which can be accessed free at:

http://rnajournal.cshlp.org/content/13/12/2141.full.html\#ref-list-1

\section{License}

Email Alerting Receive free email alerts when new articles cite this article - sign up in the box at the Service top right corner of the article or click here. 